\title{
Research on Big Data and Artificial Intelligence Aided Decision-Making Mechanism with the Applications on Video Website Homemade Program Innovation
}

\author{
Ting Li \\ Wuhan Donghu University, No.301, Wenhua Ave., \\ Jiangxia District,Wuhan,Hubei 430212,P.R.China
}

\begin{abstract}
In this paper, we conduct research on the big data and the artificial intelligence aided decision-making mechanism with the applications on video website homemade program innovation. Make homemade video shows new media platform site content production with new possible, as also make the traditional media found in Internet age, the breakthrough point of the times. Site homemade video program, which is beneficial to reduce copyright purchase demand, reduce the cost, avoid the homogeneity competition, rich advertising marketing at the same time, improve the profit pattern, the organic combination of content production and operation, complete the strategic transformation. On the basis of these advantages, once the site of homemade video program to form a brand and a higher brand influence. Our later research provides the literature survey for the related issues.
\end{abstract}

Keywords- Bid Data, Artificial Intelligence, Decision-Making, Video Website, Program Innovation.

\section{Introduction}

Since the beginning of the video website industry in China has been plagued by poor quality of the content, copyright infringement, imperfect service system. Country has enacted a number of laws and regulations on the administrative intervention, specification in order to ensure the normal operation, but any strong administrative interference and can only solve the media business at that time the pain, media in a market economy can't be replaced in their own survival and development for a long time. Under the huge pressure of competition, the only way for the development of video web site for a long time is to implement the brand strategy, to adapt to the macro environment, to ensure the survival and development of website. At present, China's video website industry market competition is fierce the only way for its long development is to implement basic brand strategy, brand building through to in multiple transmission channel of the competition to ensure the survival and development of website.

Network variety show is in the network movies, TV series after exploration and formed the scale of a new category for websites are investing in self entertainment, for the following reasons. (1) Under existing conditions for high cost of television series copyright purchase video website is regardless of the net cost of inputs, and causing thousands of network side of the boat and homemade program can not only meet the demand of users, more can highlight the features and form their own brand style as also implemented at the same time, video website from platform to platform and the transformation of content producers. (2) Network variety show is a new kind of product in the past two years, it is each website after micro film and network homemade plays, another to seek new development product. In a broad sense, refers to the video website with perfect technical platform support, to provide Internet users online smooth release, view and share the video works website. 
(3) Compared with television, the video site also has the function of storage can view the content of the past, the first phase of the program can also by clicking on the progress bar for selective watch, also need not stand ads during the show, watch the way more freedom and with the development of tablet computers, mobile phones and other mobile terminals popularization, the audience can through these watching fast and mobile terminals, for the network variety show the batch of young viewers [1-3].

With the wide application of database technology and the high demand for information in today's society, people with transaction processing as core, supporting the business operating environment and platform of the database technology has already cannot adapt to the needs of the people in analysis and decision-making level. In order to effectively for the enterprise management and decision-making process provides important information, need according to the decision need to collect relevant data from enterprise inside and outside, and properly handle, in order to form an integrated environment for decision making. Data mining technology is as core independent of information technology. Data mining is the analysis of the data in the database, data warehouse, and obtains a series of methods and techniques useful knowledge and information. Abroad, the data mining technology has developed rapidly, and gradually become a new means of decision support. Correspondingly, in the figure one, we show the features of the decision-making mechanism with data [4-5].

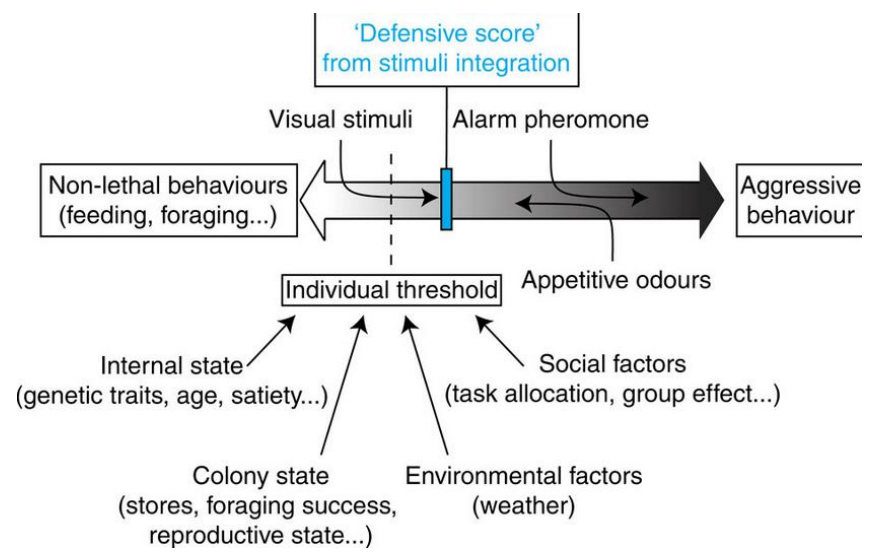

Figure 1. The Demonstration of the Decision-Making Mechanism

In this paper, we conduct research on the big data and artificial intelligence aided decision-making mechanism with the applications on video website homemade program innovation. Along the way, video website between points lot of new changes have taken place in modern marketing competition, business model is more and more diversified, network linkage, copyright, content of self-control and so on more than one competition that has formed the complete marketing pattern, based on the video website competition as the breakthrough point to discuss the marketing model.

\section{The Proposed Methodology}

The Development of the Big Data. Nowadays, global data volumes are growing at an unprecedented rate, and with the expansion of the global wireless network coverage, and the emergence of the mobile devices and the development, the growth of the data is no longer restricted by time and place. For now, the large amount of data accumulation and growth mainly experienced three stages.

Since the industrial revolution, humans pay more attention to the role of data as different industry successively identified data standards, 
and accumulated a lot of structured data, the rise of computer and network the emergence of large amounts of data analysis query processing technology makes the efficient processing of large amounts of traditional structured data possible. For the challenges of the big data systems, we could summarize them as the follows. (1) Problem of big data analyzing and processing. Distributed computing, the parallel computing can provide effective support, and how to effectively use the existing distributed, parallel technology to carry out the analysis of large data processing to study. (2) Big data acquisition problems. How big data has shrunk, in the case of no loss of value as far as possible to reduce the size of the data, like data cleaning, removal, etc., namely how to effectively deal with the large data similar to the effect of physics, the large data size smaller but not loss value. (3) Big data storage problem. For structured data, data query, statistics, update efficiency is low for unstructured data, such as pictures, video, file storage, retrieval, such as difficult [6].

As the result of the information technology rapid development, emphasized the separation of data and operation data, using big data technology are in conformity with the current management system to achieve huge amounts of data on demand and according to the right of sharing data is the core of the various industries and departments of competitiveness, more and more get the attention of industry sector, three technology, seven points according to, fully reflects the current industry's attitude to data.

\section{The Artificial Intelligence Decision.} Moment in the rapid development of information science and technology of the today, senior leaders have to make major decisions become more and more difficult, in order to solve this problem, the intelligent decision support system as an important aspect in the field of management information system, has become a new hot topic in the field of computer management application and important research direction. Development, construction and use of the intelligent decision support system to enhance the ability of knowledge development and utilization, improve the intelligent decision-making level to improve the effect of the application of information system has important theoretical significance and practical value [7-8].

With the deepening of the research and application, artificial intelligence technology develops very fast. Now, artificial intelligence has gradually formed such as basic expert system, machine learning, pattern recognition, the natural language understanding, robotics, game, artificial neural network, and many other research areas. (1) System is based on knowledge of artificial intelligence in the most significant contribution for industrial applications. Expert system is the problem solving ability with expert level in related field of intelligent application system as it can use the domain experts for many years of accumulated experience and expertise, simulating the thinking process of human experts, to solve the need to human experts to solve the problem. (2) Artificial neural network. The term artificial neural network, which is relative to the biology of the biological neural network system and it is the purpose of the participant with a simple mathematical model for biological neural network structure is described, and in certain algorithm on the premise of the guidance, make its can simulate biological neural network to some of extent with the intelligent behaviors, with the traditional algorithm of the intelligent information processing problems. (3) Decision support system is a new edge discipline, its application is a kind of innovation of the management technology into productive forces, it involves statistics, operations research, knowledge acquisition, data visualization and basic high performance computing, expert system, behavior science, systems theory and some other related disciplines of the knowledge. Decision knowledge is in to the system data based on detailed insight, analysis and the speculation, so the biggest difficulty lies in the incomplete information and specification, and 
nature of the data are often uncertain, and the people is according to the incomplete, non-standard, uncertain information to make the decisions, so the data statistical processing is an important part.

The Characteristics of the Video Websites. The rise of video web site is the product of various environment fusion from the perspective of the bigger, can be divided into the policy environment, economic environment, technology environment three aspects. It is the three aspects of environment, video website underway in China's rise in the earth.

China's gross domestic product maintained good and stable growth, for the domestic development of various undertakings to provide a solid foundation. In addition to overall economic environment in our country, the following two aspects are crucial to the development of the video website. (1) The all-round development of the Internet industry for the development of video website provides a good economic environment. After ten years of development, China's Internet has already formed the scale, Internet applications to diversification. The Internet is more and more profoundly changing people's study, work and lifestyle, and even affects the entire social process. (2) Market investors have video web sites. According to a survey of the Internet society of China, 2006 podcasts and video website user scale in our country has reached 76 million. This is a must for many investors to seize the chance and the later development of video website also confirmed the rationality of this prediction.

Content homogeneity, innovation is insufficient. Due to the video web site to make homemade requires a lot of money, and these programs from generation to mature to launch needs a certain time period, so the video website of homemade program represents a small part of all the video web sites. And a larger proportion which is from the traditional media and the supply of professional film and television production companies, so the video website video repeat rate is high, did not find their proper positioning, many simply rely on introduction of popular TV dramas to attract users. Channel video site homogeneity serious basic as drama, movies, variety, anime, original channel columns, lead to channel does not have its own characteristic, does not have its own unique attraction, and video website for update of the site is limited to page itself is beautiful and perfect, is not the deep reform, therefore, most of the video website video mode and video interface is also very similar.

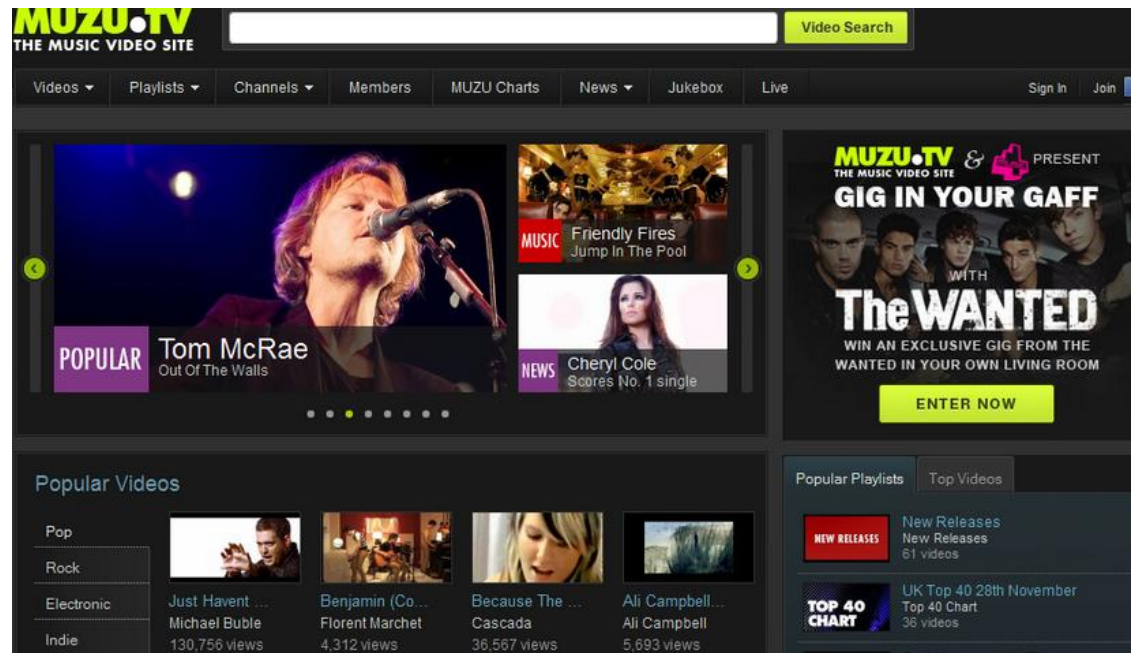

Figure 2. The Illustration of the Foreign Video Website Homemade Program Form 
As shown in the figure two, for the video website development for long time and stable operation, the introduction of the need to increase funding. At the same time, due to the development of science and technology and the rise of the emerging media has its unique mode of the transmission, and its contents form also has more advantages than traditional media. Video web site, therefore, have to do is make use of these advantages, reorganizes video website profit pattern research project was carried out on the complicated and changeable market, forming a set of suitable for the existing video website market operation pattern [9].

The Modern Internet Video Innovation. Industrial innovation today media industry to maintain a competitive advantage in the increasingly competitive environment, the basic way of along with the continuous development of media technology, at the same time, both of the economic and ideological attribute media group must through continuous reform and innovation to gain the ability to survive. Under this background, we suggest the listed suggestions for the related programs.

- As the improvement of network bandwidth, network video technology continue to improve, the media industry in innovation, as a strategic priority must be the video innovation, as the key to promoting the construction of the media as the key to grasp. More and more customers will transfer the product promotion focus to a more open network platform, the media industry want to survive and gain a competitive advantage that must actively use new technology.

- Social platform with interactive mode of technical means, greatly shorten distance between media and the public, the media industry in the reform and innovation have a broader feedback channels, through the use of the interactive strong WeChat, microblogging, blog, not just for media workers get more fresh and up-to-date news, more able to communicate with audience.

- Along with the social media in a short period of time in ear trend worldwide, social network as the core of rapid development of Internet, traditional media marketing has also been a impact.

- Media technology progress, makes the gap between different communication means more and more small, even has a tendency to melt, each big media, especially the traditional plane media, must actively use modern methods of the media through the use of the mobile platform and build a form such as electronic newspapers to attract more and more readers at the same time, create a more diverse business model, to ensure its own competitive power and to create the necessary premise to ensure competitive advantage.

\section{Conclusion}

In this paper, we conduct research on the big data and the artificial intelligence aided decision-making mechanism with the applications on the video website homemade program innovation. As the newly emerging thing, the development status quo of network entertainment is enough to make the video website. Make money less, simple manufacturing process, but can get so much benefit, brand value for video website sets up the armband. Although the present network entertainment programs exists some problems, but there is no doubt that the development of it found a new way for the video sites. Network entertainment programs will be as the basic demand of the audience, making more and more sophisticated, the path of a benign development and believe it will be video website of the trend of the times. In the future, we will conduct more in-depth analysis on the issues. 


\section{References}

[1] Monstad, Therese, and Stefan Hrastinski. "An interactive video website as a mode of communication in a participatory process." (2014).

[2] Braun, Michael T. "Obstacles to social networking website use among older adults." Computers in Human Behavior 29.3 (2013): 673-680.

[3] Liang, Ying, and Hanrong Chen. "The research of video resource personalized recommendation system based on education website." Computer Science \& Education (ICCSE), 2014 9th International Conference on. IEEE, 2014.

[4] Ferguson, Christopher J. "Violent video games and the Supreme Court: lessons for the scientific community in the wake of Brown v. Entertainment Merchants Association." American Psychologist 68.2 (2013): 57.
[5] Brooks, Sarah Jeanne. Adoption of technological innovations: A Case Study of the ASSESS website. Diss. WASHINGTON STATE UNIVERSITY, 2013.

[6] Maddison, Ralph, et al. "Active video games: the mediating effect of aerobic fitness on body composition." Int J Behav Nutr Phys Act 9.1 (2012): 54.

[7] Scott, David Meerman. The new rules of marketing \& PR: How to use social media, online video, mobile applications, blogs, news releases, and viral marketing to reach buyers directly. John Wiley \& Sons, 2013.

[8] Munaretto, Daniele, et al. "Online path selection for video delivery over cellular networks." Globecom Workshops (GC Wkshps), 2012 IEEE. IEEE, 2012.

[9] Vishwakarma, Sarvesh, and Anupam Agrawal. "A survey on activity recognition and behavior understanding in video surveillance." The Visual Computer 29.10 (2013): 983-1009. 